\title{
About the Mass
}

\section{Angel Fierros Palacios}

División de Energías Alternas, Instituto de Investigaciones Eléctricas, Cuernavaca Morelos, México

Email: afierros@iie.org.mx

How to cite this paper: Palacios, A.F. (2017) About the Mass. Open Access Library Journal, 4: e2835.

https://doi.org/10.4236/oalib.1102835

Received: June 18, 2017

Accepted: August 8, 2017

Published: August 11, 2017

Copyright $@ 2017$ by author and Open Access Library Inc.

This work is licensed under the Creative Commons Attribution International License (CC BY 4.0).

http://creativecommons.org/licenses/by/4.0/

\begin{abstract}
In this paper it is proposed that the mass of the bodies has its origin and nature in the reciprocal gravitational interactions between them; and also by some kind of effect over the size of the celestial bodies due to the very big distances in space, as seemed each other at a distance. In a Dynamic Theory ot Gravitation [1], it is proved that the fundamental velocity is the escape velocity due to the apparent size of the interacting heavenly bodies, which is the medium used by gravity to transmit its effects like propagating force of Nature [2]. Given that is the greatest speed of the Universe, the celestial bodies interact between them in a reciprocal way [3]. Because of that dynamical process all those bodies have an intrinsic property called mass. Then, the mass of any body is a kind of parameter by means of which a measure of the inertial effects can be obtained. That property is different from weight. It is a consequence of the gravitational interactions between any body and all the rest of the heavenly bodies of the Universe, and also by some deep characteristic of the space that separate them.
\end{abstract}

\section{Subject Areas}

Classical Mechanics

\section{Keywords}

Escape Velocity, Gravitational and Inertial Mass, Weight

\section{Introduction}

It was Galileo who discovered that bodies fall at a rate independent of their mass. He used as a tools an incline plane to slow the fall, a water clock to measure the time, and a pendulum to avoid rolling friction [4]. Newton could use his second law to conclude that the force exerted by gravity over a body is proportional to its mass [5]. However, he was aware that his conclusions might be approximately true; in such a way that the inertial mass of his second law might not be the same 
as the gravitational mass appearing in the law of gravitation [5]. The question of the equality of both kinds of masses is linked to the problem of the concept of mass as a deep property of the bodies, those of gravity, and also the very big distances in space.

\section{Weight and Mass}

The weight of a body is the gravitational force exerted on it by a source of the gravity attraction. Being a force, is a vector whose direction is the direction of the gravity force; that is, toward the center of the source [5]. When a body of mass $m$ is allowed to fall freely on the Earth's surface, its acceleration is the gravity acceleration $\boldsymbol{g}$, and the force acting on it is its weight $W$.

Newton's second law

$$
\boldsymbol{F}=m \boldsymbol{a},
$$

where $F$ is the applied force, $m$ the mass, and $\boldsymbol{a}$ the acceleration, when is applied to a freely falling body, takes the following form

$$
W=m \boldsymbol{g} .
$$

where both vectors are directed toward the center of the Earth; so that it can therefore write that

$$
W=m g \text {; }
$$

$W$ and $g$ are the magnitudes of the weight and acceleration vectors, respectively.

To keep an object from falling, it has to exert on it an upward force equal in magnitude to $W$ so as to make the net force zero [5]. It is well known that $g$ is found experimentally to have the same value for all bodies at the same place [5]. From this it follows that the ratio of the weights of two objects must be equal to the ratio of their masses [5]. Therefore a balance, which is an instrument for comparing downward forces, can be used in practice to compare masses. However, it is important to distinguish carefully between weight and mass, because the weight of a body is a vector quantity, in the meantime its mass is a scalar quantity [5]. Besides, the weight is measured with the body at rest, and the mass of the same body is measured in movement.

The relationship between weight and mass is given by the equation (2). Because $g$ varies from point to point on the Earth, $\boldsymbol{W}$ which is the weight of a body of mass $m$, is different in different localities. Hence, unlike the mass of a body which is an intrinsic property of the body, the weight depends on its location relative to the center of the Earth. Then, the weight of a body is zero in regions of space where the gravitational field or its effects, are null; although the inertial effects, and hence the mass of the body remains unchanged from those on the Earth [5].

It takes the same force to accelerate a body in a gravity-free space as it does to accelerate it along a horizontal frictionless surface on the Earth; because its mass is the same in each place; but it takes a greater force to hold the body against to 
pull of the Earth on the earth's surface than it does high up in space, because its weight is different in each place.

Often, instead of being given the mass, are given the weight of the body on which the forces are exerted. The acceleration a produced by the force $F$ acting on a body whose weight is $W$ can be obtained combining the Equations (1) and (3). Therefore from $\boldsymbol{F}=m \boldsymbol{a}$ and $W=m g$ it is easy to obtain that

$$
m=\frac{W}{g} \text { and } \boldsymbol{F}=\left(\frac{W}{g}\right) \boldsymbol{a},
$$

The quantity $W / g$ plays the role of $m$ in the Equation (1), and is in fact the mass of the body whose weight is $W$ [5].

\section{Gravitational and Inertial Mass}

If the inertial mass of Newton's second law were not the same as the gravitational mass in the law of gravitation, it would have to write the Newton's second law as

$$
\boldsymbol{F}=m_{i} \boldsymbol{a}
$$

and write the law of gravitation as

$$
\boldsymbol{F}=m_{g} \boldsymbol{g}
$$

where $\boldsymbol{g}$ is the gravity acceleration which is a field depending on position and other masses [4]. Thus, the acceleration at a given point would be

$$
\boldsymbol{a}=\left(\frac{m_{g}}{m_{i}}\right) \boldsymbol{g} ;
$$

and would be different for bodies with different values of the ratio $m_{g} / m_{i}$. In particular, pendulums of equal length would have periods proportional to $\left(m_{i} / m_{g}\right)^{1 / 2}$. Newton in experiments with pendulums of equal length but different composition tested that possibility and found no differences in their periods [4]. That result was later verified more accurately by F.W. Bessel [4], and also by R.v. Eötvos [6] [7] [8], who proved by a different method that the ratio does not differ from one material to another by more than one part in $10^{9}$. A few years later, a group under R.H. Dicke [9] [10] improved on Eötvos method by using the gravitational field of the Sun, and Earth's centripetal acceleration toward the Sun, rather than the rotation of the Earth, to produce a torque on the balance of the Eötvos experiment [4]. The conclusion was that the aluminum and gold fall toward the Sun with the same acceleration differing each other by at most one part in $10^{11}$ [9] [10]. It has also been shown, with very much less precision, that neutrons fall with the same acceleration as ordinary matter; and that the gravitational force on electrons in Cooper is the same as on free electrons [4].

\section{Gravity and Mass}

In spite of the experimental results by means of which the equality of the gravitational and inertial mass are proved; the question about the real nature of the 
mass has not an answer. Many teachers and books head of the question assuming that the problem of the mass, as an intrinsic property of the bodies, has already been solved. However, it is clear that the mass is independent of the material, composition, and atomic structure of the bodies; and also is not the same as the weight. So that, even today that problem is centered in the question about the origin and nature of the mass.

In Newton's Law of Universal Gravitation it is implicit the idea that the gravity force between two celestial bodies is independent of the presence of other heavenly bodies, or the properties of the space between them [5]. Nevertheless, that idea might not be true, because those bodies are not insolated in the space; in such a way that it is important to take into account the gravitational attraction by all the bodies of the Universe which is exerted over any body by means of the escape velocity due to their apparent sizes [2]. So that, when the escape velocity of any body, due to its apparent size, in some region of the space, meets with the respective escape velocity of the rest of the celestial bodies, they pull the body, but that body pull de other bodies, because those velocities due to their respective apparent sizes, are the carriers of their gravitational fields [2]. Given that the gravity force is always attractive, that dynamic process is the responsible for the link which is established between the body and the other bodies. It is proposed here that as a result of that influence the body has the intrinsic property known as its mass. So that, the mass is something produced by gravity as a deep characteristic of the bodies; in such a way that it is possible to conclude that the gravitational and inertial mass are the same thing; as it was proved experimentally by Galileo, Newton, Bessel, Eötvos, and Dicke [4].

\section{Conclusions}

It is possible to assert that the gravitational interactions between the heavenly bodies of the Universe, interact each other in a reciprocal way, and also due to their apparent sizes, as seemed at a distance are the responsible of the intrinsic property of any body known as its mass. That is to say, the gravitational influence is the origin and nature of that important physical property.

From that point of view, as it was said before, perhaps the mass is a kind of parameter by means of which a measure of the inertial effects proper of any body, can be explained.

Also, and this is very important, the reciprocal gravitational interactions play a special role in the structure of the Solar System, the Galaxies, and the Universe itself. In fact, that dynamical process is responsible of the construction of a kind of three-dimensional and complex network by means of lines of gravity force, like those of the magnetic field, which link the heavenly bodies between them as seemed each other at a distance in the space. This is so because the gravity force is always attractive.

In the reciprocal gravitational interactions [2], in order to obtain the escape velocity it is used the concept of apparent size [2]. Given that none source of 
gravitational attraction in Nature has size zero, and that the speed of gravitational interactions use as transport medium that velocity, it can be concluded that in Nature can't exist instantaneous velocities. As the speed of gravitational interactions, using as transport medium the escape velocity due to the apparent size, increases its value with distance [2], then it is concluded that necessarily there must be a maximum distance among the heavenly bodies; this fact suggests a finite Universe.

Finally, it is well known that $A$. Einstein was very impressed with the observed equality of gravitational and inertial mass, and that knowledge it can served him as a signpost towards the Principle of Equivalence [4].

\section{References}

[1] Fierros Palacios, A. (2015). The Small Deformation Strain Tensor as a Fundamental Metric Tensor. Journal of High Energy Physics, Gravitation and Cosmology, 1, 35-47. https://doi.org/10.4236/jhepgc.2015.11004

[2] Fierros Palacios, A. (2015). Gravitation. Open Access Library Journal.

[3] Fierros Palacios, A. (2015). The Reciprocity Principle in Gravitational Interactions. Open Access Library Journal.

[4] Weinberg, S. (1972) Gravitation and Cosmology. Principles and Applications of the General Theory of Relativity. John Wiley \& Sons. Inc., New York, London, Sydney, Toronto.

[5] Resnick, R. and Holliday, D. (1966) Physics. John Wiley \& Sons Inc. Hoboken.

[6] Eötvos. R.V. (1890) Math. Nat. Ber. Hungarian 65.

[7] Eötvos, R.V., Pekar, D. and Felcete, E. (1922) Ann. Phys. 68.

[8] Kenner, J. (1935) Hung. Acad. Sci., Vol. 53 Part II.

[9] Dicke, R.H., DeWitt, C. and DeWitt, B.S. (1964) Relativity Groups, and Topology, Gordon and Breach, New York, 167.

[10] Roll, P.G., Krothov, R. and Dicke, R.H. (1967) Ann Phys, New York, Vol. 26, 442.

Open Access Library

Submit or recommend next manuscript to OALib Journal and we will provide best service for you:

- Publication frequency: Monthly

- 9 subject areas of science, technology and medicine

- Fair and rigorous peer-review system

- Fast publication process

- Article promotion in various social networking sites (LinkedIn, Facebook, Twitter, etc.)

- Maximum dissemination of your research work

Submit Your Paper Online: Click Here to Submit

Or Contact service@oalib.com 